\title{
Neue Bücher aus Oberwolfach
}

Frank H. Lutz

Auf den Auslageregalen der Bibliothek in Oberwolfach fiel mir neben den vielen anderen Neuerscheinungen das Buch Experimental Mathematics von Vladimir I. Arnold (ins Englische übersetzt von Dmitry Fuchs und Mark Saul) sofort ins Auge. In vier Vorlesungen (The Statistics of Topology and Algebra, Combinatorial Complexity and Randomness, Random Permutations and Young Diagrams of Their Cycles und The Geometry of Frobenius Numbers for Additive Semigroups), die Arnold im Jahr 2005 im Dubna-MathematikSommer-Camp gehalten hat, lädt er die Leserinnen und Leser zum arithmetischen und numerischen Experimentieren ein. So lässt sich aus den Beispielen $5 \cdot 5=25$ und $6 \cdot 6=36$ offensichtlich die Vermutung $7 \cdot 7=47$ aufstellen. Arnold regt an, solche Vermutungen durch gezieltes Experimentieren zu bestätigten oder zu widerlegen. "Not having achieved what they desired, they pretended to desire what they had achieved" - mit diesem der Einleitung vorangestellten Zitat von M. Montel umreißt Arnold den in seinen Vorlesungen beschriebenen Findungsprozess. Dennoch ist das Buch keine leichte Lektüre, da Arnold oft assoziativ argumentiert und viele Zwischenschritte nicht ausführt. Ziel des Buches ist jedoch vor allem, explorativ in neues mathematisches Terrain vorzudringen und die Freude daran weiterzugeben.

Ursprünglich als Anhang zu einer Monografie von Matthias Aschenbrenner und Stefan Friedl gedacht, hat sich das Buch 3-Manifold Groups der Autoren Aschenbrenner, Friedl und Henry Wilton zu einem wahren Kleinod entwickelt. Auf 215 Seiten und mit über 1300 Literaturhinweisen versehen, ist es den Autoren gelungen, den Leserinnen und Lesern einen umfassenden und kondensierten Überblick zum derzeitigen Stand der Kenntnis zu Fundamentalgruppen von kompakten 3-Mannigfaltigkeiten an die Hand zu geben - und damit auch zu 3-Mannigfaltigkeiten selbst, da irreduzible, orientierbare, geschlossene 3-Mannigfaltigkeiten, bis auf Linsenräume, eindeutig durch ihre Fundamentalgruppe bestimmt sind. Das erste Kapitel des Buches ist der Zerlegung kompakter, orientierbarer 3-Mannigfaltigkeiten und der Geometrisierung der Bestandteile gewidmet, bevor es an die Klassifikation von 3-Mannigfaltigkeiten über ihre Fundamentalgruppen geht. In späteren Kapiteln werden insbesondere Resultate von Agol, Kahn-Markovic und Wise zu hyperbolischen 3-Mannigfaltigkeiten besprochen sowie Untergruppen von 3-Mannigfaltigkeitsgruppen. Das Buch liest sich wie ein Geschichtsbuch der 3-Mannigfaltigkeitsgruppen, in dem sich alle wichtigen Entwicklungen, Sätze und Spezialfälle klar strukturiert finden lassen. Abgerundet wird dieser Leitfaden des Gebiets durch ein präzises Einflechten der relevanten Literaturhinweise. Selbst die einseitige Abgrenzung ,What this book is not about" (Fundamentalgruppen von nicht-kompakten 3-Mannigfaltigkeiten, Fun- damentalgruppen von speziellen Klassen von 3-Mannigfaltigkeiten etc.) ist wiederum mit einer Vielzahl an Literaturverweisen versehen, sodass man sich wünschen möchte, einen weiteren „Anhang“ der Autoren zu diesen Themen lesen zu können. Ach ja, die Autoren verweisen darauf, dass ihr Werk nicht als Erstlektüre zu 3Mannigfaltigkeiten gedacht ist, sondern dass Grundkenntnisse bereits voraussetzt werden.

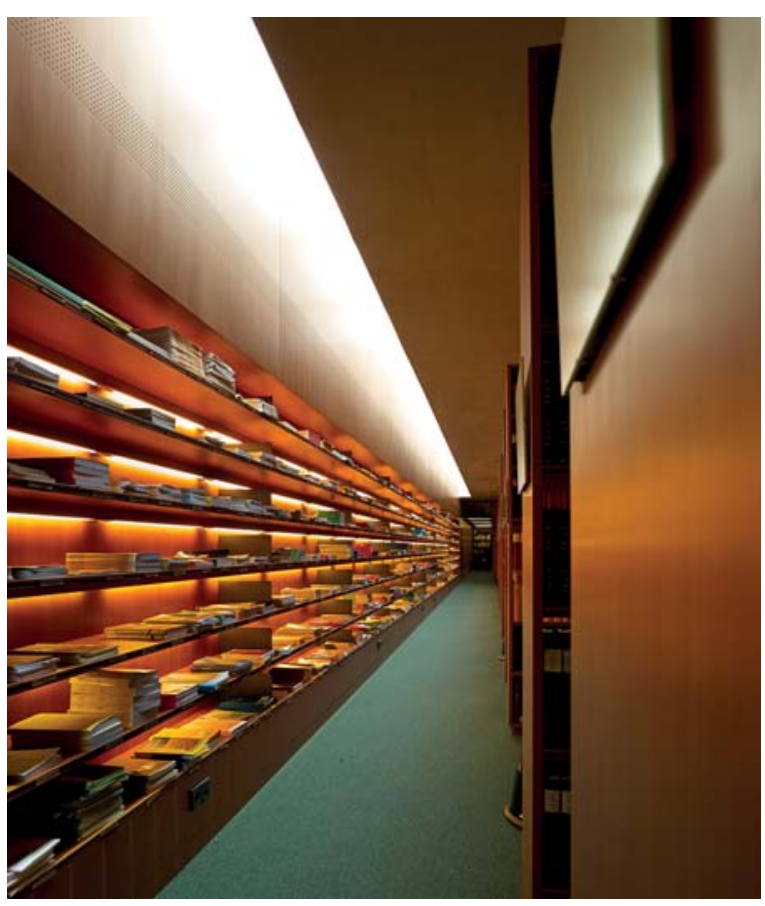

In der Bibliothek des MFO (Foto: Christoph Eyrich)

Als Start in die 3-Mannigfaltigkeiten bietet sich das Buch Introduction to 3-Manifolds von Jennifer Schultens an. Zielgruppe sind vorwiegend Studierende, weshalb auf einen möglichst elementaren Einstieg Wert gelegt wird. Hierzu betrachtet Schultens 3-Mannigfaltigkeiten aus unterschiedlichen Perspektiven: topologische Mannigfaltigkeiten, differenzierbare Mannigfaltigkeiten, orientierbare Mannigfaltigkeiten, triangulierte Mannigfaltigkeiten, geometrische Mannigfaltigkeiten, und diskutiert Äquivalenzen der Kategorien. Die wesentlichen Themen im Hauptteil des Buches sind dann Flächen, Zerlegungen von 3-Mannigfaltigkeiten in irreduzible Bestandteile, Knoten und Zöpfe, Triangulierungen, Heegaard-Zerlegungen und hyperbolische Mannigfaltigkeiten. Inhaltlich umfangreich, mit vielen Aufgaben versehen und angenehm zu lesen, ist das Buch für einen Einstieg sehr zu empfehlen.

PD Dr. Frank Lutz, Institut für Mathematik, MA 5-2, Technische Universität Berlin, Straße des 17. Juni 136, 10623 Berlin lutz@math.tu-berlin.de 